\title{
Effect of Laval Nozzle in the GAMMA 10 SMBI Experiments*)
}

\author{
Md. Maidul ISLAM, Yousuke NAKASHIMA, Shinji KOBAYASHI ${ }^{1)}$, Nobuhiro NISHINO ${ }^{2)}$, \\ Yuichiro NAKANO ${ }^{1)}$, Katsuhiro HOSOI, Kazuya ICHIMURA, Md. Shahinul ISLAM, \\ Keita SHIMIZU, Kazuma FUKUI, Masato OHUCHI, Akihiro TERAKADO, \\ Masayuki YOSHIKAWA, Junko KOHAGURA, Mafumi HIRATA, Ryuya IKEZOE, \\ Xiaolong WANG, Makoto ICHIMURA, Mizuki SAKAMOTO and Tsuyoshi IMAI \\ Plasma Research Center, University of Tsukuba, Tsukuba, Ibaraki 305-8577, Japan \\ ${ }^{1)}$ Institute of Advanced Energy, Kyoto University, Gokasyo, Uji 611-0011, Japan \\ ${ }^{2)}$ Graduate School of Engineering, Hiroshima University, Hiroshima 739-8527, Japan
}

(Received 30 November 2015 / Accepted 3 March 2016)

Gas fueling by supersonic molecular beam injection (SMBI) has been performed at the central-cell in GAMMA 10. The results of SMBI experiment with plenum pressure from 0.3 MPa to 2.0 MPa were obtained using a laval nozzle. The distribution of the emission intensity during SMBI was investigated by the 2-dimensional image captured by the fast camera as an index of the neutral transport. The experimental results indicates that the effect of the laval nozzle reduces the dispersion of injected hydrogen molecules in the peripheral region. In this paper, we also studied the line density at the central-cell (NLcc) and $\mathrm{H} \alpha$ emission intensity during SMBI. Comparison between SMBI and conventional gas puffing is also discussed from the view point of the fueling efficiency.

(C) 2016 The Japan Society of Plasma Science and Nuclear Fusion Research

Keywords: GAMMA 10, supersonic molecular beam injection, Laval nozzle, $\mathrm{H} \alpha$, high-speed camera

DOI: $10.1585 /$ pfr. 11.2402053

\section{Introduction}

For future thermonuclear fusion reactors fueling is an important issue. Fueling control enables the profile control of the core plasma density and reduction in neutral particles in the peripheral area. Pellet injection (PI) can reduce the edge recycling and help to obtain a peak density profile, which is important for obtaining better confinement [1]. PI has become a standard and powerful refueling method, after more than 20 years of enthusiastic effort. Although this system is suitable for device ITER-like tokamaks, the system is complicated and it is not easy to make a pellet small enough for density control in medium or small devices. On the other hand, supersonic molecular beam injection (SMBI) developed by L. Yao et al. [2], is suitable for future ITER-like tokamaks as well as small device like GAMMA 10 [3-7]. SMBI is economical to develop and to maintain. SMBI gives high-speed and high-directive gas injection because of higher plenum pressure compared to the conventional gas puffing and it can inject neutral particles deeper into the core plasma.

GAMMA 10 is the world largest tandem mirror and is an open magnetic plasma-confining device $[8,9]$. The central-cell has a simple solenoidal magnetic configuration with ten pancake coils. In addition, GAMMA 10 has many observation ports at the central-cell. Thus, the central-cell

author'se-mail: maidul@prc.tsukuba.ac.jp

*) This article is based on the presentation at the 25 th International Toki Conference (ITC25). is suitable for analyzing the plasma behavior during SMBI. SMBI port is located at the bottom of the central-cell midplane. The first experimental results of SMBI by straight nozzle showed that SMBI achieved higher density plasmas at the core region than the conventional gas puffing [6]. In this paper we have carried out SMBI experiments by using the laval nozzle. The purpose of this study is to investigate the neutral particles behavior during SMBI by using the laval nozzle. Comparison between straight and laval nozzle is also discussed from the view of neutral dispersion.

\section{GAMMA 10 and Experimental Setup}

A schematic view of GAMMA 10 tandem mirror together with heating and diagnostic system related to the fueling experiments is shown in Fig. 1. The total length of GAMMA 10 is $27 \mathrm{~m}$, and volume of the vacuum vessel is $150 \mathrm{~m}^{3}$. The vacuum vessel consists of centralcell, anchor-cells, plug/barrier-cells and end-cells. The main confinement region of GAMMA 10 is the central cell $(Z=-300 \sim+300 \mathrm{~cm})$. The initial plasma produced by the plasma gun is heated by ICRF waves and confined by magnetic mirror field and electrostatic potential. Two choke coils and ten pancake coils are installed and formed the simple axi-symmetric magnetic mirror configuration in the central-cell. The both ends of central cell are connected to east/west anchor-cells, which keep the 


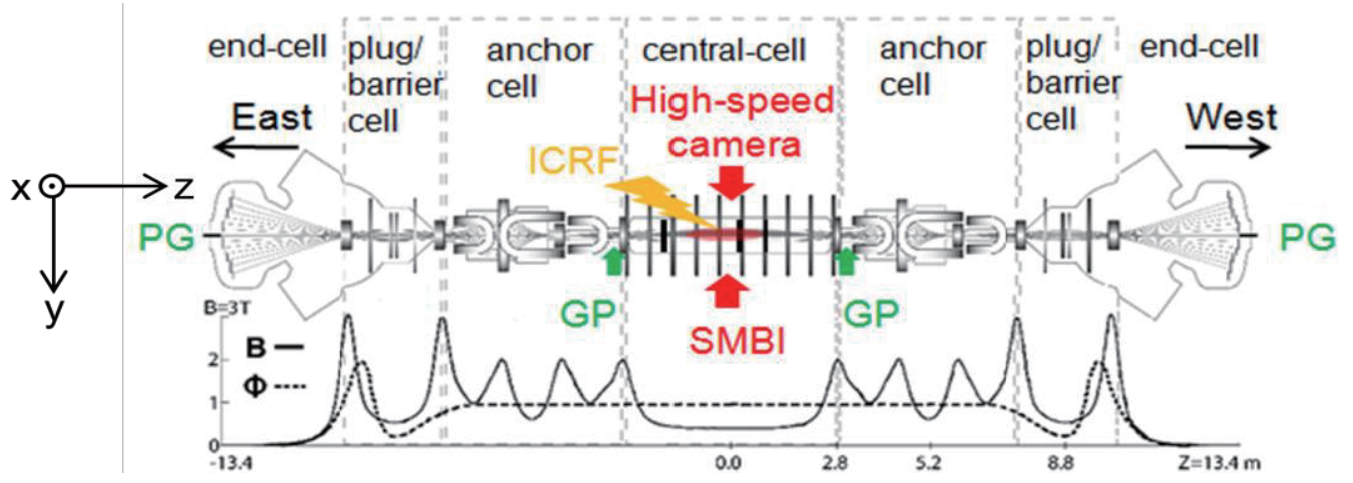

Fig. 1 Schematic view of GAMMA 10 and axial profile of magnetic field strength $B$ and potential $\phi$. The locations of Experimental tools are shown.

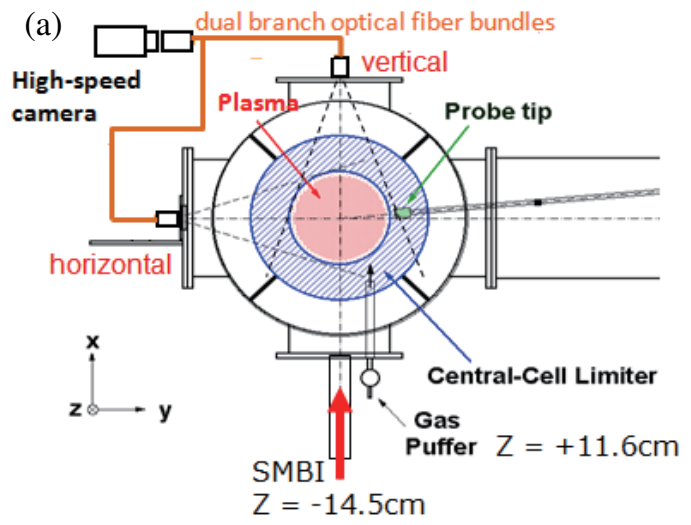

(b)

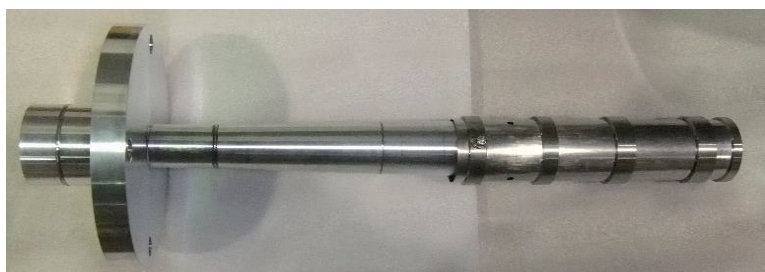

Fig. 2 (a) Cross-sectional view of the central-cell, locations of SMBI system and high-speed camera, (b) Photograph of the laval nozzle.

plasma stably. Three baseball coils are installed and form the average minimum-B configuration in each anchorcell. The outside of anchor-cells are east/west plug/barriercells, in which plug potential and barrier potential are produced. East/west end-cells are located to the outside of plug/barrier-cells in which the plasma flow out.

Figure 2 shows the cross-section view of the GAMMA 10 central-cell and the optical arrangement for fast camera, and the sketch of the SMBI system and photograph of laval nozzle. The SMBI system consists of a fast solenoid valve with a magnetic shield. In GAMMA 10, SMBI system with laval nozzle has been installed in the central-cell at $Z=-14.5 \mathrm{~cm}$. The conventional gas puffing fueling system is also located in the central-cell at $Z=+11.6 \mathrm{~cm}$. A high-speed camera has been installed at the central-cell in order to observe the emission from the plasma. The highspeed camera detects the 2-dimensional $(x-z$, or $y-z)$ image of visible light emission from the plasma during SMBI. The camera system has dual blanch optical fiber bundles, in which two lines of sight can be taken at the same time at horizontal and vertical direction of the plasma crosssection. In Fig. 2 (b) the total view of the laval nozzle is shown. The total length of the laval nozzle is $363 \mathrm{~mm}$ and the diameter of throat and exit sections are $0.6 \mathrm{~mm}$ and $24 \mathrm{~mm}$, respectively.

To investigate the neutral behavior at the central-cell, three $\mathrm{H} \alpha$ detector has been installed at $Z=-1 \mathrm{~cm}, Z=$ $-71 \mathrm{~cm}$ and $Z=-141 \mathrm{~cm}$. Each detector consists of an $\mathrm{H} \alpha$ filter, an optical fiber and a photomultiplier. The detected signal of $\mathrm{H} \alpha$ line-emission is transferred to a CAMAC system and finally analyzed with a workstation. In our experiment, the plenum pressure is varied from $0.3 \mathrm{MPa}$ to 2.0 $\mathrm{MPa}$ and the pulse width is usually $0.5 \mathrm{~ms}$.

\section{Experimental Results and Discus- sion}

In the experiment, using laval nozzle SMBI pulses were injected into the typical plasmas heated by only ICRF. A typical 2-D image captured by the fast camera of visible emission during SMBI with laval nozzle from the plasma is shown in Fig. 3. This 2-D image was captured at the peak emission intensity. It took $1.2 \mathrm{~ms}$ to achieve the peak intensity after the SMBI. It indicates SMBI with laval nozzle is faster than that with straight nozzle $(2 \mathrm{~ms})$ [6]. The left side of Fig. 3 shows the intensity emitted in the vertical direction; the right side shows that emitted in the horizontal direction. The 2-D image viewing from the horizontal port is more widely spread than the diameter of the nozzle exit. The injected molecules are dissociated with Frank-Condon energy $(\sim 3 \mathrm{eV})$ much higher than molecular velocity (thermal energy). It is considered that the dissociated hydrogen atoms spread out as isotropic and resultant emission profile becomes much wider than that of original beam. To 


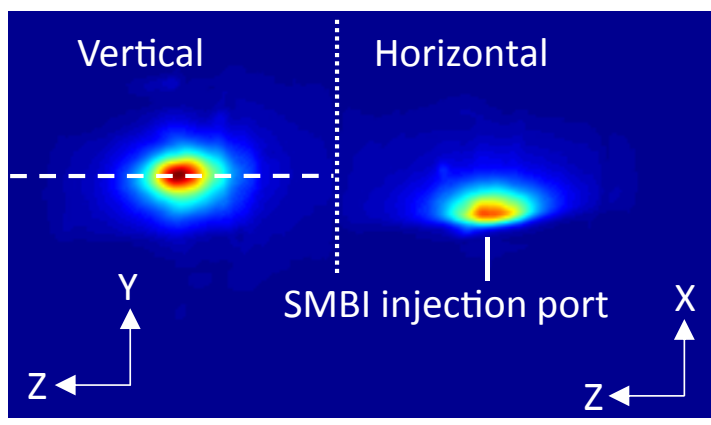

Fig. 3 Two-dimensional image during SMBI.

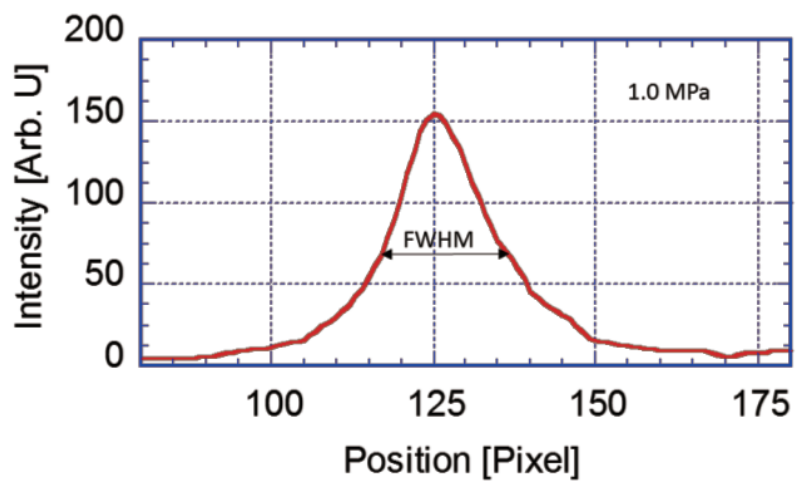

Fig. 4 Distribution of vertical emission intensity.

evaluate the axial profile of neutral transport, the directivity of the molecular beam injected by SMBI is investigated based on vertical direction image. We also studied the distribution of emission intensity at the broken line on the left side of Fig. 3 (The broken line was drawn over the SMBI injection port). Figure 4 shows the distribution of emission intensity at the broken line as obtained from the 2-D vertical image.

We used the full width at half maximum (FWHM) of the distribution of the emission intensity at the broken line on the left side of Fig. 3 as an index of the axial neutral transport [4]. As the intensity profile generally does not change as the intensity increase, the best signal-to-noise ratio is obtained at the peak emission intensity. Using the laval nozzle we analyzed the FWHM for each SMBI plenum pressure and compared the FWHM obtained by a straight nozzle. All experimental conditions except for the SMBI plenum pressure were fixed.

Figure 5 shows the relationship between FWHM and the plenum pressure for both nozzle. Remarkable reduction of FWHM is achieved using laval nozzle. Moreover, in all plenum pressure FWHM is lower than the diameter of plasma. Accordingly, the effect of the laval nozzle reduces the spreading of the neutral particle from SMBI in the peripheral region. The FWHM value decreases with increasing the SMBI plenum pressure in both cases. In low plenum pressure the flow of molecular beam is thought to be not supersonic. The FWHM decrease with increasing

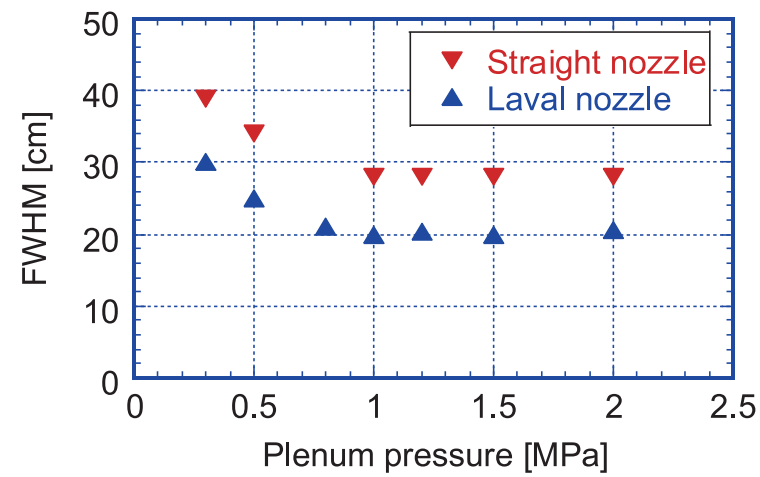

Fig. 5 Variation of FWHM with plenum pressure for both nozzle.

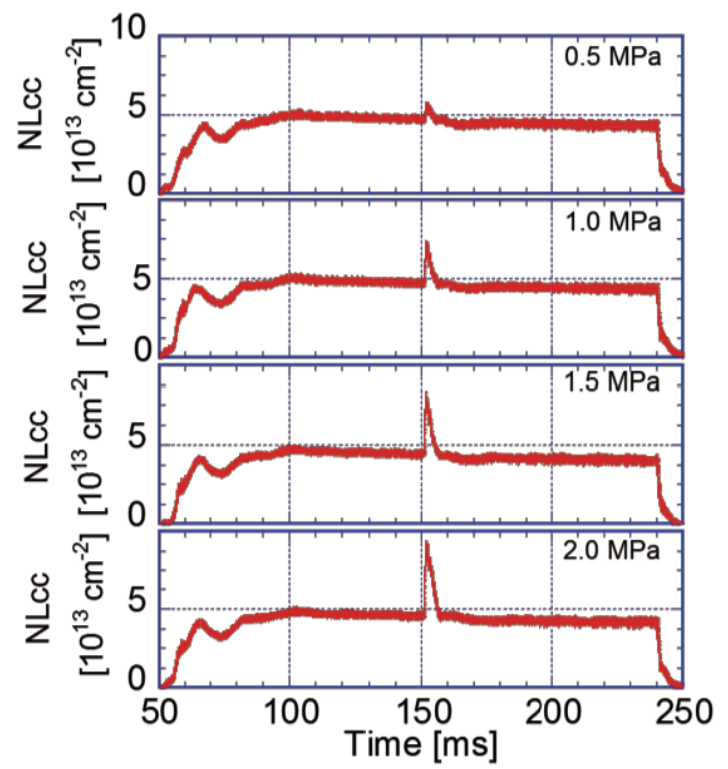

Fig. 6 Electron line density in the central-cell during SMBI.

plenum pressure up to $1.0 \mathrm{MPa}$ and then the flow become supersonic. At high plenum pressure (1.0 MPa or higher) FWHM is saturated it could be due to the convergence of molecular beam.

Figure 6 shows how the plasma parameter electron line density (NLcc) varies during SMBI at different plenum pressure. At pressure $0.5 \mathrm{MPa}$ the line density is low because amount of gas at this pressure is less and particles spreads in the peripheral region. However, when the pressure is $1.0 \mathrm{MPa}$ or higher, the line density was increased quickly, since at this pressure the amount of gas is high as well as reduces the diffusion in the peripheral region as we mentioned.

Figure 7 shows $\mathrm{H} \alpha$ emission intensity during SMBI at the plenum pressure of 1.0 MPa. It is observed that the emission intensity at $Z=-1 \mathrm{~cm}$ is high, since the $\mathrm{H} \alpha$ detector at this position is close to the SMBI injection port. The response of SMBI is decreased with increasing distance from the SMBI injection position, since the particles injected at this pressure become more convergent. 


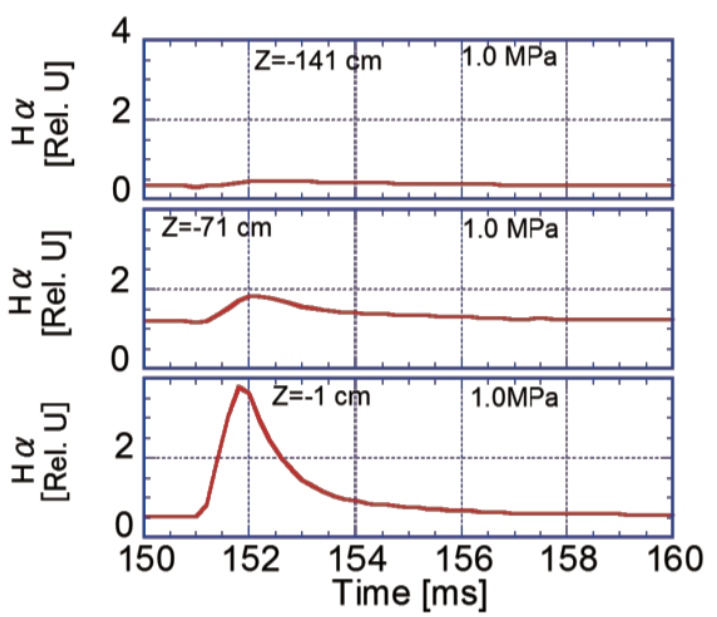

Fig. $7 \mathrm{H} \alpha$ emission intensity during SMBI.

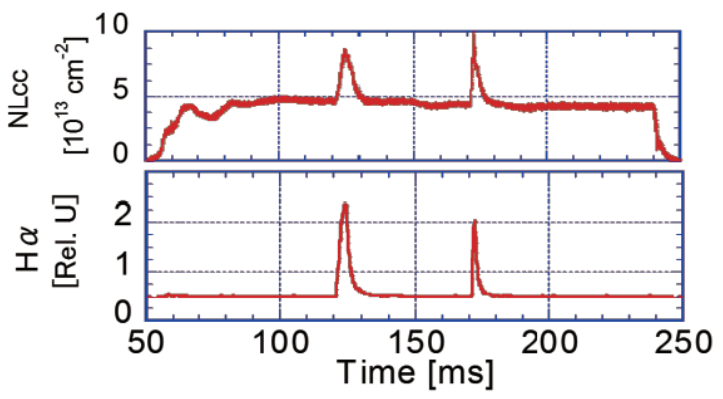

Fig. 8 Comparison of plasma parameters in between GP and SMBI experiments.

The comparison between conventional gas puffing (GP) and SMBI is shown in Fig. 8. GP and SMBI are injected at $120 \mathrm{~ms}$ and $170 \mathrm{~ms}$, durations are $4 \mathrm{~ms}$ and $1 \mathrm{~ms}$, and pressures are $66.66 \mathrm{kPa}$ and $1.0 \mathrm{MPa}$, respectively. Increased portion of NLcc is higher during SMBI than that of gas puffing. On the other hand, the peak value of the $\mathrm{H} \alpha$ intensity measured from the same detector at $Z=-1 \mathrm{~cm}$, is lower and the time width become narrower during SMBI than gas puffing. It qualitatively shows that SMBI with laval nozzle is more efficient than gas puffing. However detail comparison of the fueling rate is our future task.

In GAMMA 10, multi-channel microwave interferometer has been installed at central-cell to observe the radial profile of line-integrated density at core and peripheral region. The radial profile of electron density for fueling and without fueling as shown in Fig. 9. Both fueling system are effecting at core and peripheral region. It is found that the electron density is slightly higher in the case of SMBI than gas puffing.

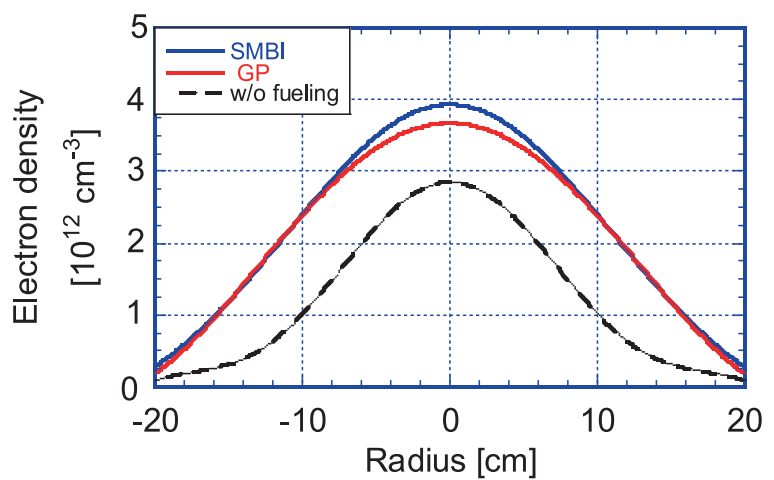

Fig. 9 Radial profile of electron density during gas puffing and SMBI.

\section{Summary}

We investigate the properties of neutral transport from SMBI with laval nozzle. The FWHM is lower than the straight nozzle, which indicated the Laval nozzle reduces the dispersion of the neutral particle from SMBI in the peripheral region. However, the FWHM was almost saturated in the plenum pressure range of more than $1 \mathrm{MPa}$. It may be caused by the convergence of molecular beam. The NLcc is higher during SMBI than the gas puffing. On the other hand, the $\mathrm{H} \alpha$ intensity is low and become steeper during SMBI than the gas puffing. It means SMBI with laval nozzle is more efficient than gas puffing.

In future, we investigate the effect of laval nozzle by simulation and compare with experimental results.

\section{Acknowledgments}

This study was supported by the bilateral collaboration research program in the University of Tsukuba, Kyoto University, and Hiroshima University (NIFS12KUGM068, NIFS12KUHL050 and NIFS12KUGM065). The authors would like to thank the members of the GAMMA 10 group fort their collaboration in the experiments and for helpful discussion.

[1] R. Sakamoto et al., Nucl. Fusion 41, 381 (2001).

[2] L. Yao et al., Nucl. Fusion 47, 1399 (2007).

[3] R. Pegourie et al., J. Nucl. Mater. 313-316, 539 (2003).

[4] K. Hosoi et al., Plasma Fusion Res. 9, 3402087 (2014).

[5] A. Murakami et al., Plasma Phys. Control. Fusion 54, 055006 (2012).

[6] K. Hosoi et al., Trans. Fusion Technol. 63, 244 (2013).

[7] T. Mizuuchi et al., Contrib. Plasma Phys. 50, No.6-7, 639 (2010).

[8] M. Inutake et al., Phys. Rev. Lett. 55, 939 (1985).

[9] Y. Nakashima et al., J. Nucl. Mater. 415, S996 (2011). 